PROCEEDINGS OF THE

AMERICAN MATHEMATICAL SOCIETY

Volume 133, Number 9, Pages 2721-2727

S 0002-9939(05)07813-5

Article electronically published on March 22, 2005

\title{
LOW REGULARITY SOLUTIONS FOR A CLASS OF NONLINEAR WAVE EQUATIONS
}

\author{
NIKOLAOS BOURNAVEAS
}

(Communicated by Christopher D. Sogge)

\begin{abstract}
We construct local low regularity solutions for a class of nonlinear wave equations with power-type nonlinearities.
\end{abstract}

\section{INTRODUCTION}

The purpose of this note is to present a new proof of the existence of local low regularity solutions to the Cauchy problem

$$
u_{t t}-\Delta u=F(u), u(0, \cdot)=f, u_{t}(0, \cdot)=g
$$

in the case of three space dimensions and for nonlinearities $F(u)$ which 'behave like' a 'supercritical' power $u^{p}, p>5$ (see Theorem 2.3 for precise assumptions on $F$ ). We assume that $f \in H^{s}\left(\mathbb{R}^{3}\right)$ and $g \in H^{s-1}\left(\mathbb{R}^{3}\right)$ with $s=\frac{3}{2}-\frac{2}{p-1}$ (critical). This result was first proved by Lindblad and Sogge [LS using Strichartz estimates.

Applying the standard energy estimate to the wave equation in (1.1) we obtain the term $\int_{0}^{T}\|F(u(t))\|_{H^{s-1}} d t$, which is the same, roughly speaking, as $\int_{0}^{T}\left\|u^{p-1}(t) \partial^{s-1} u(t)\right\|_{L_{x}^{2}} d t$ and can be estimated by $\int_{0}^{T}\|u(t)\|_{L_{x}^{\infty}}^{p-1}\left\|\partial^{s-1} u(t)\right\|_{L_{x}^{2}} d t$. In the classical theory of nonlinear wave equations the Sobolev inequality is used to estimate the $L_{x}^{\infty}$ norm, thus imposing the restriction $s>\frac{3}{2}$. However, a simple scaling argument shows that the critical value of $s$ is $s=\frac{3}{2}-\frac{2}{p-1}$. In [LS] (see also chapter 3 of $[\mathbf{S}$ ) the authors prove a Strichartz-type estimate which, when applied to (1.1), produces a term which behaves like $\left\|\partial^{s-1 / 2} u^{p}\right\|_{L^{4 / 3\left([0, T] \times \mathbb{R}^{3}\right)}}$, and this space-time norm is then estimated using another Strichartz estimate. In this argument the energy estimate is not used and the $L^{\infty}$ norm of the solution doesn't come up at all.

On the other hand, as Klainerman has pointed out in $[\mathrm{K}]$, in the case $p=5, s=1$, the term $\int_{0}^{T}\left\|u^{5}(t)\right\|_{L_{x}^{2}} d t$ which the energy estimate brings in can be estimated by $\left(\int_{0}^{T}\|u(t)\|_{L_{x}^{\infty}}^{2} d t\right)^{1 / 2} \cdot\|u\|_{L^{8}\left([0, T] \times \mathbb{R}^{3}\right)}^{4}$. The second factor is exactly what the standard Strichartz estimate can handle and, in the first factor, the square of the $L^{\infty}$ norm is integrated in time. The relevant estimate here is the "missing Strichartz

Received by the editors September 1, 2003 and, in revised form, May 3, 2004.

2000 Mathematics Subject Classification. Primary 35L70.

(C)2005 American Mathematical Society Reverts to public domain 28 years from publication 
inequality',

$$
\left(\int_{0}^{T}\|\phi(t)\|_{L^{\infty}\left(\mathbb{R}^{3}\right)}^{2} d t\right)^{1 / 2} \leq C\left[\left\|\phi_{0}\right\|_{H^{1}\left(\mathbb{R}^{3}\right)}+\left\|\phi_{1}\right\|_{L^{2}\left(\mathbb{R}^{3}\right)}\right]
$$

where $\phi$ solves $\phi_{t t}-\Delta \phi=0$ with initial data $\left(\phi_{0}, \phi_{1}\right) \in H^{1} \oplus L^{2}$. As it turns out, this estimate is true in the spherically symmetric case but fails in general [KM, $[\mathrm{M}-\mathrm{S}] \mathrm{S}]$. If, however, the initial data have $\epsilon$ derivatives more, namely $\phi_{0} \in H^{1+\epsilon}\left(\mathbb{R}^{3}\right)$, $\phi_{1} \in H^{\epsilon}\left(\mathbb{R}^{3}\right)$, then certain space-time norms of the form $\left(\int_{0}^{T}\|\phi(t)\|_{L_{x}^{\infty}}^{l} d t\right)^{1 / l}$ can actually be estimated: a Sobolev embedding can be used to control $\|\phi(t)\|_{L_{x}^{\infty}}$ by $\left\|\partial^{\epsilon} \phi(t)\right\|_{L_{x}^{p}}$, where $p$ is suitably large, followed by a Strichartz-type estimate to control the resulting mixed space-time norm. This idea, implicit in the proof of the main theorem of $[\mathrm{PS}]$, was also used in $[\mathrm{B}]$ and was later developed systematically in [EV] where the end-point case we shall need was proved (see Lemma 2.1). In this end-point case the Sobolev embedding alluded to above fails and a more delicate argument is needed. We aim to show in this note that estimates involving spacetime integrals of the $L^{\infty}$ norm can be very useful in the study of equation (1.1), and that, at least in the case $p>5$, the energy estimate need not be avoided. Our argument is a modified version of Klainerman's observation discussed above.

Notation. We denote by $H_{p}^{s}$ the standard Sobolev spaces in $\mathbb{R}^{3}$. Mixed spacetime norms are defined by

$$
\|u\|_{L_{t}^{r} L_{x}^{p}}=\left(\int_{0}^{T}\left(\int_{\mathbb{R}^{3}}\|u(t, x)\|^{p} d x\right)^{\frac{r}{p}} d t\right)^{\frac{1}{r}} .
$$

Unlabelled norms refer to the space variable $x$. We use obvious abbreviated notation as, for example, $\int_{0}^{T}\|u\|_{L^{\infty}}^{p-1}\left\|\partial^{s-1} u\right\|_{L^{2}} d t$, which stands for

$$
\int_{0}^{T}\|u(t, \cdot)\|_{L^{\infty}\left(\mathbb{R}^{3}\right)}^{p-1}\|u(t, \cdot)\|_{H^{s-1}\left(\mathbb{R}^{3}\right)} d t .
$$

The wave operator is defined by $\square=\partial_{t}^{2}-\Delta$.

\section{LOCAL EXISTENCE}

The Strichartz-type estimate that we shall need is contained in the following.

Lemma 2.1. Let $f \in H^{s}\left(\mathbb{R}^{3}\right), g \in H^{s-1}\left(\mathbb{R}^{3}\right), F \in L^{1}\left(I ; H^{s-1}\left(\mathbb{R}^{3}\right)\right)$ and let $\phi$ solve:

$$
\phi_{t t}-\Delta \phi=F, \phi(0, \cdot)=f, \phi_{t}(0, \cdot)=g .
$$

Assume $s \in\left(1, \frac{3}{2}\right)$ and define $l=\frac{1}{\frac{3}{2}-s}$. Then there is a positive constant $C$ such that

$$
\left(\int_{I}\|u(t, \cdot)\|_{L_{x}^{\infty}\left(\mathbb{R}^{3}\right)}^{l} d t\right)^{1 / l} \leq C\left[\|f\|_{H^{s}}+\|g\|_{H^{s-1}}+\int_{I}\|F(t, \cdot)\|_{H^{s-1}\left(\mathbb{R}^{3}\right)} d t\right] .
$$

Here $I$ is either $(0, \infty)$ or any interval of the form $(0, T)$ with $T>0$. In the second case, $C$ is independent of $T$.

Proof. See estimate (1.14b) in [EV]. This may be seen as the end-point case of estimate $(1.14 \mathrm{a})$ in that paper. 
We shall also use the following 'fractional chain rule'.

Lemma 2.2. Let $F$ be a smooth real-valued function, $p, q, r \in(1, \infty)$ and $s \in(0,1)$ with $\frac{1}{r}=\frac{1}{p}+\frac{1}{q}$. Then for any $u \in L^{\infty}\left(\mathbb{R}^{3}\right) \cap \dot{H}_{q}^{s}\left(\mathbb{R}^{3}\right)$ we have

$$
\|F(u)\|_{\dot{H}_{r}^{s}\left(\mathbb{R}^{3}\right)} \leq C\left\|F^{\prime}(u)\right\|_{L^{p}\left(\mathbb{R}^{3}\right)}\|u\|_{\dot{H}_{q}^{s}\left(\mathbb{R}^{3}\right)} .
$$

Proof. See Proposition 25, Chapter III in [C] and also [CW].

With these two tools at hand we can now prove our main result.

Theorem 2.3. Let $f \in H^{s}\left(\mathbb{R}^{3}\right), g \in H^{s-1}\left(\mathbb{R}^{3}\right)$ where $s=s_{c r}=\frac{3}{2}-\frac{2}{p-1}$ and $p>5$ (equivalently: $s>1$ ). Let $F$ be a smooth real-valued function such that $\|F(u)\| \leq C\|u\|^{p}$ and $\left\|F^{\prime}(u)\right\| \leq C\|u\|^{p-1}$. Then there is a $T>0$, depending only on the initial data $f$ and $g$, such that the Cauchy problem

$$
u_{t t}-\Delta u=F(u), u(0, \cdot)=f, u_{t}(t, \cdot)=g
$$

has a unique solution

$$
u \in C^{0}\left([0, T] ; H^{s}\left(\mathbb{R}^{3}\right)\right) \cap C^{1}\left([0, T] ; H^{s-1}\left(\mathbb{R}^{3}\right)\right)
$$

with

$$
\int_{0}^{T}\|u(t, \cdot)\|_{L^{\infty}}^{\frac{p-1}{2}} d t<\infty .
$$

If, moreover, the quantity $D_{s}=\|f\|_{H^{s}}+\|g\|_{H^{s-1}}$ is sufficiently small, then the solution exists globally in time.

Proof. Fix $T \in(0,1)$ and $M>0$ to be determined later. Define the $s$-energy

$$
E_{s}(u)=\sup _{0 \leq t \leq T}\left[\|u(t, \cdot)\|_{H^{s}\left(\mathbb{R}^{3}\right)}+\left\|u_{t}(t, \cdot)\right\|_{H^{s-1}\left(\mathbb{R}^{3}\right)}\right]
$$

and the modified $s$-energy

$$
E_{s}^{m}(u)=\sup _{0 \leq t \leq T}\left[\|u(t, \cdot)-f\|_{H^{s}\left(\mathbb{R}^{3}\right)}+\left\|u_{t}(t, \cdot)-g\right\|_{H^{s-1}\left(\mathbb{R}^{3}\right)}\right] .
$$

Observe that $E_{s}(u) \leq E_{s}^{m}(u)+D_{s}$, where $D_{s}=\|f\|_{H^{s}}+\|g\|_{H^{s-1}}$, and also that $E_{s}^{m}\left(u_{1}\right) \leq E_{s}\left(u_{1}-u_{2}\right)+E_{s}^{m}\left(u_{2}\right)$. Define

$$
|u|_{s}=E_{s}^{m}(u)+\left(\int_{0}^{T}\|u\|_{L_{x}^{\infty}}^{\frac{p-1}{2}} d t\right)^{\frac{2}{p-1}} .
$$

Let $w$ be the solution of the linear Cauchy problem,

$$
w_{t t}-\Delta w=0, w(0, \cdot)=f, w_{t}(0, \cdot)=g .
$$

By linear theory,

$$
E_{s}^{m}(w) \rightarrow 0 \quad, \quad \text { as } T \rightarrow 0 .
$$

By (2.1), and since $\frac{p-1}{2}=\frac{1}{\frac{3}{2}-s}$, we have $\int_{0}^{\infty}\|w(t, \cdot)\|_{L^{\infty}}^{\frac{p-1}{2}} d t<\infty$; hence

$$
\int_{0}^{T}\|w(t, \cdot)\|_{L^{\infty}}^{\frac{p-1}{2}} d t \rightarrow 0 \quad, \quad \text { as } T \rightarrow 0 .
$$

Therefore,

$$
|w|_{s} \rightarrow 0 \quad, \quad \text { as } T \rightarrow 0 .
$$


Define

$$
X=\left\{u \in C^{0}\left([0, T] ; H^{s}\left(\mathbb{R}^{3}\right)\right) \cap C^{1}\left([0, T] ; H^{s-1}\left(\mathbb{R}^{3}\right)\right):|u|_{s} \leq M\right\} .
$$

We equip $X$ with the metric

$$
d\left(u_{1}, u_{2}\right)=\sup _{0 \leq t \leq T}\left[\left\|u_{1}(t, \cdot)-u_{2}(t, \cdot)\right\|_{H^{1}\left(\mathbb{R}^{3}\right)}+\left\|\partial_{t} u_{1}(t, \cdot)-\partial_{t} u_{2}(t, \cdot)\right\|_{L^{2}\left(\mathbb{R}^{3}\right)}\right] .
$$

Then $(X, d)$ is a complete metric space. Consider the map $\mathcal{T}: X \rightarrow X$ defined as follows: Given $u \in X, v=\mathcal{T}(u)$ is the unique solution of the linear Cauchy problem

$$
v_{t t}-\Delta v=F(u), v(0, \cdot)=f, v_{t}(0, \cdot)=g .
$$

We first need to prove that $\mathcal{T}$ maps $X$ into $X$. So let $u \in X$ and $v=\mathcal{T}(u)$. Then

$$
|v|_{s} \leq|w|_{s}+E_{s}(v-w)+\left(\int_{0}^{T}\|v-w\|_{L^{\infty}}^{\frac{p-1}{2}} d t\right)^{\frac{2}{p-1}} .
$$

Now $v$ and $w$ have the same initial data and $\square(v-w)=F(u)$. Therefore, by the energy estimate and the Strichartz estimate (2.1),

$$
E_{s}(v-w)+\left(\int_{0}^{T}\|v-w\|_{L^{\frac{p-1}{2}}} d t\right)^{\frac{2}{p-1}} \leq C \int_{0}^{T}\|F(u)\|_{H^{s-1}\left(\mathbb{R}^{3}\right)} d t .
$$

We have

$$
\int_{0}^{T}\|F(u)\|_{H^{s-1}} d t \leq C \int_{0}^{T}\|F(u)\|_{L^{2}} d t+C \int_{0}^{T}\|F(u)\|_{\dot{H}^{s-1}} d t:=I+I I .
$$

To estimate $I I$ we first use the fractional Leibniz rule (2.2) to get

$$
\begin{aligned}
I I & \leq C \int_{0}^{T}\left\|F^{\prime}(u)\right\|_{L^{3}}\|u\|_{\dot{H}_{6}^{s-1}} d t \\
& \leq\left. C E_{s}(u) \int_{0}^{T}\|\| u\right|^{p-1} \|_{L^{3}} d t \\
& \leq C E_{s}(u) \int_{0}^{T}\|u\|_{L^{\infty}}^{\frac{p-1}{2}}\|u\|_{L^{3(p-1) / 2}}^{\frac{p-1}{2}} d t .
\end{aligned}
$$

Next, since $\frac{3(p-1)}{2}=\frac{6}{3-2 s}$ we have $\|u(t, \cdot)\|_{L^{3(p-1) / 2}} \leq C\|u(t, \cdot)\|_{H^{s}} \leq E_{s}(u)$; hence,

$$
\begin{aligned}
I I & \leq C E_{s}(u)^{1+\frac{p-1}{2}} \int_{0}^{T}\|u\|_{L^{\infty}}^{\frac{p-1}{2}} d t \\
& \leq C\left(E_{s}^{m}(u)+D_{s}\right)^{\frac{p+1}{2}}|u|_{s}^{\frac{p-1}{2}} \\
& \leq C\left(|u|_{s}+D_{s}\right)^{\frac{p+1}{2}}|u|_{s}^{\frac{p-1}{2}} .
\end{aligned}
$$


To estimate the integral $I$ observe that $p+1 \leq \frac{3(p-1)}{2}=\frac{6}{3-2 s}$; hence by Sobolev, $\|u(t, \cdot)\|_{L^{p+1}} \leq C\|u(t, \cdot)\|_{H^{s}} \leq E_{s}(u)$. Therefore,

$$
\begin{aligned}
I & \leq C \int_{0}^{T}\left\||u|^{p}\right\|_{L^{2}} d t \\
& \leq C \int_{0}^{T}\|u\|_{L^{\infty}}^{\frac{p-1}{2}}\|u\|_{L^{p+1}}^{\frac{p+1}{2}} d t \\
& \leq C E_{s}(u)^{\frac{p+1}{2}} \int_{0}^{T}\|u\|_{L^{\infty}}^{\frac{p-1}{2}} d t \\
& \leq C\left(E_{s}^{m}(u)+D_{s}\right)^{\frac{p+1}{2}}|u|_{s}^{\frac{p-1}{2}} \\
& \leq C\left(|u|_{s}+D_{s}\right)^{\frac{p+1}{2}}|u|_{s}^{\frac{p-1}{2}} .
\end{aligned}
$$

Therefore,

$$
\int_{0}^{T}\|F(u)\|_{H^{s-1}} d t \leq C\left(|u|_{s}+D_{s}\right)^{\frac{p+1}{2}}|u|_{s}^{\frac{p-1}{2}} .
$$

From (2.5), (2.6) and (2.8),

$$
|v|_{s} \leq|w|_{s}+C\left(|u|_{s}+D_{s}\right)^{\frac{p+1}{2}}|u|_{s}^{\frac{p-1}{2}} .
$$

Since $u \in X$ we have $|u|_{s} \leq M$. Therefore,

$$
|v|_{s} \leq|w|_{s}+C\left(M+D_{s}\right)^{\frac{p+1}{2}} M^{\frac{p-1}{2}} .
$$

Thanks to (2.4) we can choose a sufficiently small $T$ so that

$$
C\left(2|w|_{s}+D_{s}\right)^{\frac{p+1}{2}}\left(2|w|_{s}\right)^{\frac{p-1}{2}} \leq|w|_{s} .
$$

Set $M=2|w|_{s}$. Then

$$
|v|_{s} \leq|w|_{s}+C\left(M+D_{s}\right)^{\frac{p+1}{2}} M^{\frac{p-1}{2}} \leq \frac{M}{2}+\frac{M}{2}=M
$$

We have shown that $|v|_{s} \leq M$ whenever $u \in X$. We have also shown (see estimate (2.8)) that $v_{t t}-\Delta v=F(u) \in L^{1}\left([0, T] ; H^{s-1}\right)$. It follows from linear theory that $v \in C^{0}\left([0, T], H^{s}\left(\mathbb{R}^{3}\right)\right) \cap C^{1}\left([0, T], H^{s-1}\left(\mathbb{R}^{3}\right)\right)$. This completes the proof that $v \in X$ whenever $u \in X$.

Now let $u_{1}, u_{2} \in X$. Then, by the standard energy estimate,

$$
\begin{aligned}
d\left(\mathcal{T}\left(u_{1}\right), \mathcal{T}\left(u_{2}\right)\right) \leq & C \int_{0}^{T}\left\|F\left(u_{1}\right)-F\left(u_{2}\right)\right\|_{L^{2}} d t \\
\leq & C \int_{0}^{T}\left\|\left(u_{1}-u_{2}\right)\left(\left|u_{1}\right|+\left|u_{2}\right|\right)^{p-1}\right\|_{L^{2}} d t \\
\leq & C \int_{0}^{T}\left\|u_{1}-u_{2}\right\|_{L^{6}}\left\|u_{1}\right\|_{L^{3(p-1) / 2}}^{\frac{p-1}{2}}\left\|u_{1}\right\|_{L^{\infty}}^{\frac{p-1}{2}} d t \\
& +\int_{0}^{T}\left\|u_{1}-u_{2}\right\|_{L^{6}}\left\|u_{2}\right\|_{L^{3(p-1) / 2}}^{\frac{p-1}{2}}\left\|u_{2}\right\|_{L^{\infty}}^{\frac{p-1}{2}} d t .
\end{aligned}
$$

By Sobolev inequalities,

$$
\left\|u_{1}(t, \cdot)-u_{2}(t, \cdot)\right\|_{L^{6}} \leq C d\left(u_{1}, u_{2}\right)
$$

and

$$
\left\|u_{1,2}(t, \cdot)\right\|_{L^{3(p-1) / 2}} \leq C\left|u_{1,2}\right|_{s} \leq C M .
$$


Therefore,

$$
\begin{aligned}
d\left(\mathcal{T}\left(u_{1}\right), \mathcal{T}\left(u_{2}\right)\right) \leq & C M^{\frac{p-1}{2}} d\left(u_{1}, u_{2}\right) \int_{0}^{T}\left\|u_{1}\right\|_{L^{\infty}}^{\frac{p-1}{2}} d t \\
& +C M^{\frac{p-1}{2}} d\left(u_{1}, u_{2}\right) \int_{0}^{T}\left\|u_{2}\right\|_{L^{\infty}}^{\frac{p-1}{2}} d t \\
\leq & C M^{p-1} d\left(u_{1}, u_{2}\right) .
\end{aligned}
$$

Recall that $M=2|w|_{s} \rightarrow 0$ as $T \rightarrow 0$. Therefore, if $T$ is sufficiently small, we have a contraction. Uniqueness is proved using similar estimates.

Suppose now that the quantity $D_{s}=\|f\|_{H^{s}}+\|g\|_{H^{s-1}}$ is small. The precise smallness condition will be determined in the course of the proof. Instead of proving that the solution we have just constructed exists globally in time, it is easier to construct directly a global solution. For this purpose we define the 'homogeneous' $s$-energy by

$$
\dot{E}_{s}(u)=\sup _{0 \leq t<\infty}\left[\|u(t, \cdot)\|_{\dot{H}^{s}\left(\mathbb{R}^{3}\right)}+\left\|u_{t}(t, \cdot)\right\|_{\dot{H}^{s-1}\left(\mathbb{R}^{3}\right)}\right]
$$

and the space

$$
X=\left\{u \in C^{0}\left([0, \infty) ; H^{s}\left(\mathbb{R}^{3}\right)\right) \cap C^{1}\left([0, \infty) ; H^{s-1}\left(\mathbb{R}^{3}\right)\right):|u|_{\dot{s}} \leq M\right\}
$$

where

$$
|u|_{\dot{s}}=\dot{E}_{s}^{m}(u)+\left(\int_{0}^{T}\|u\|_{L_{x}^{\frac{p-1}{2}}}^{\frac{p}{2}} d t\right)^{\frac{2}{p-1}}
$$

and $M$ will be determined later. Estimates similar to those leading to (2.7) can be employed to show that

$$
|v|_{\dot{s}} \leq C D_{s}+C|u|_{\dot{s}}^{p} .
$$

We then define $M=2 C D_{s}$ and make the smallness assumption $C M^{p} \leq \frac{M}{2}$, equivalently $D_{s} \leq\left(\frac{1}{2 C}\right)^{\frac{p}{p-1}}$. With this choice of $M$ we then have

$$
|v|_{\dot{s}} \leq C D_{s}+C|u|_{\dot{s}}^{p} \leq \frac{M}{2}+\frac{M}{2}=M .
$$

The rest of the proof is similar to what we have already discussed.

Remarks. 1. The condition $p>5$ is needed to ensure that $s_{c r}>1$ so that Lemma 2.1 applies.

2. The integrability condition (2.3) could be removed from the uniqueness part of the theorem using the methods of $[\mathrm{Ka}, \mathrm{P}]$.

\section{REFERENCES}

[B] Bournaveas, N. Local existence for the Maxwell-Dirac equations in three space dimensions. Comm. Partial Differential Equations 21 (1996), no. 5-6, 693-720. MR1391520 (97f:35176)

[C] Christ, M.: Lectures on singular integral operators. CBMS Regional Conference Series in Mathematics, 77, AMS, 1990. MR1104656 (92f:42021)

[CW] Christ, F. M.; Weinstein, M. I. Dispersion of small amplitude solutions of the generalized Korteweg-de Vries equation. J. Funct. Anal. 100 (1991), no. 1, 87-109. MR1124294 (92h:35203)

[EV] Escobedo, M.; Vega, L.: A semilinear Dirac equation in $H^{s}\left(R^{3}\right)$ for $s>1$. SIAM J. Math. Anal. 28 (1997), no. 2, 338-362. MR 1434039|(97k:35239)

[Ka] Kato, T. On nonlinear Schrödinger equations, II. $H^{s}$ solutions and uncontitional well posedness, J. d'Analyse Math. 67 (1995), 281-306. MR.1383498 (98a:35124a) 
[K] Klainerman, S. Mathematical theory of classical fields and general relativity. Mathematical Physics, X (Leipzig, 1991), 213-236, Springer, Berlin, 1992. MR1386408

[KM] Klainerman, S.; Machedon, M. Space-time estimates for null forms and the local existence theorem. Comm. Pure Appl. Math. 46 (1993), no. 9, 1221-1268. MR.1231427 (94h:35137)

[LS] Lindblad, H.; Sogge, C.: On existence and scattering with minimal regularity for semilinear wave equations. J. Funct. Anal. 130 (1995), no. 2, 357-426. MR.1335386 (96i:35087)

[M-S] Montgomery-Smith, S. J. Time decay for the bounded mean oscillation of solutions of the Schrödinger and wave equations. Duke Math. J. 91 (1998), no. 2, 393-408. MR 1600602 (99e:35006)

[P] Planchon, F.; On uniqueness for semilinear wave equations. Math. Z. 244 (2003), no. 3, 587-599. MR1992026 (2004e:35157)

[PS] Ponce, G; Sideris, T.C. Local regularity of nonlinear wave equations in three space dimensions. Comm. Partial Differential Equations 18 (1993), no. 1-2, 169-177. MR1211729 (95a:35092)

[S] Sogge, C.D. Lectures on nonlinear wave equations. Monographs in Analysis, II. International Press, Boston, MA, 1995. MR.1715192 (2000g:35153)

School of Mathematics, University of Edinburgh, Edinburgh EH9 3JZ, United KingDOM

E-mail address: N.Bournaveas@ed.ac.uk 